\title{
Stability and Variable Admittance Control in the Physical Interaction with a Mobile Robot
}

\author{
Regular Paper
}

\author{
Hongbo Wang ${ }^{1,2}$, Federico Patota ${ }^{3}$, Gabriele Buondonno ${ }^{3}$, Markus Haendl${ }^{4}$, \\ Alessandro De Luca $^{3}$ and Kazuhiro Kosuge ${ }^{2 *}$
}

1 School of Mechanical Engineering, Northwestern Polytechnical University, Xi'an, Shaanxi, China
2 Department of Bioengineering and Robotics, Tohoku University, Sendai, Japan
3 Dipartimento di Ingegneria Informatica, Automatica e Gestionale, Sapienza Università di Roma, Rome, Italy
4 Institute of Automatic Control, Technische Universität München, München, Germany
*Corresponding author(s) E-mail: kosuge@m.tohoku.ac.jp

Received 12 March 2015; Accepted 16 August 2015

DOI: $10.5772 / 61313$

() 2015 Author(s). Licensee InTech. This is an open access article distributed under the terms of the Creative Commons Attribution License (http://creativecommons.org/licenses/by/3.0), which permits unrestricted use, distribution, and reproduction in any medium, provided the original work is properly cited.

\begin{abstract}
Admittance controllers have been widely implemented in physical human/robot interaction (pHRI). The stability criteria and the parameter adaptation methods for admittance control have been well-studied. However, the established methods have mainly focused on human/ manipulator interaction, and cannot be directly extended to mobile robot-based pHRI, in which the nonlinearity cannot be cancelled by feedback linearizations and the measurements of the relative human/robot position and orientation are usually lacking. In this paper, we study the pHRI between a human user and a mobile robot under admittance control. We develop a robotic system which can measure the relative chest/ankle positions of the human user with respect to the robot. Using the measured human position, a human frame admittance controller is proposed to remove the nonlinearity in the system dynamics. Based on the human-frame admittance control, a stability criterion is derived. By using a human arm stiffness estimator along with the derived stability criterion, a stiffness-based variable admittance controller is designed. The effectiveness of the proposed methods in improving the pHRI
\end{abstract}

performance is tested and supported by simulations and experimental results.

Keywords Physical Human/Robot Interaction, Admittance Control, Mobile Robot, Stiffness Estimation

\section{Introduction}

In the near future, robots are expected to cooperate with humans in the same shared workspaces in industrial and domestic applications. Therefore, pHRI is attracting increasing attention in robotics research [1]. Various types of robots have been created to physically interact with humans, including serial/parallel manipulators [2 - 8], mobile robots [9 - 11], biped robots [12], and aerial robots [13]. Among the robots created for pHRI, a mobile robot (platform) has its unique advantages in its large workspace, reliable stability during movement, and long battery life; mobile robots have been applied in applications including assembly assistance [14], walking support [15] and entertainment $[16,17]$. 
To enable the robot to behave compliantly with the force/ torque arising in pHRI, two control schemes-namely impedance control and admittance control [18] - have been introduced ${ }^{1}$. The impedance controller, which requires a specially designed back-drivable mechanical structure, is often used in haptic displays [20]. In contrast, the admittance controller fits the commonly-used manipulators and mobile robots that have high gear ratios [21], and it has been widely implemented in pHRI [4, 19, 22 - 33].

Theoretically, the coupled dynamics between a passive human and a robot under admittance control preserve such passivity as well as stability. However, in practice, the dynamics of other components (e.g., a low-pass filter as in [26]) or the time discretization [34] may give rise to instabilities. Therefore, the pHRI stability is often subject to the human arm's stiffness and the admittance parameters. To find appropriate admittance parameters that stabilize the pHRI for a given stiffness, the stability criteria for admittance controllers have been well-studied, especially in human/manipulator interaction [26, 27, 30, 33, 35].

Besides human/manipulator interaction, admittance controllers have also been applied in the physical interactions between humans and mobile robots [25, 32]. However, despite the success of their applications, the stability of human/mobile robot interactions has been investigated less frequently, and the established stability criteria for manipulators cannot be directly applied to mobile robots; for a manipulator, although its dynamics are usually nonlinear, when low-level control (e.g., using feedback linearizations) in the joint space is implemented, the translational motions of the end-effector have linear reponses to external forces [36]. However, the interactions between humans and mobile robots inevitably involve rotations that introduces nonlinearity. Consequently, the analysis of pHRI stability requires a non-trivial effort in formulating a Lyapunov function. Moreover, we will show that the techniques in formulating stability criteria for manipulators cannot be easily extended to mobile robots.

Besides stability, another issue in designing admittance controllers is pHRI performance. We could use very conservative admittance parameters (e.g., a very high virtual viscosity) to ensure pHRI stability under any uncertain stiffness; however, this high viscosity would also place an extra physical burden on the human user and it thus results in poor pHRI performance. To mitigate the trade-off between stability and performance, many researchers have proposed implementing a stiffness estimator in human/manipulator interaction and recommended adjusting the admittance parameters online according to the estimated human arm stiffness [19, 26, 27, 37]. Hereafter, we refer to this method as "stiffness-dependent variable admittance control" (SDVAC).
A stiffness estimator takes in two inputs: the force/torque in pHRI and the relative position between the human and the robot. In human/manipulator interaction, since the base of the manipulator is fixed and the human operator is often standing or sitting, the change of their relative position is simply the end-effector displacement, which can easily be obtained by the joint sensors and the forward kinematics. However, in the mobile robot case, as both the human and the robot are moving, their relative position and orientation are not equal to the displacement of the robot. Although in the previous literature laser rangefinders (LRFs) have been used to measure the human/robot distances for fallprevention $[32,10]$, more detailed human information (i.e., the position and orientation of the human body) is still lacking. Consequently, the lack of human pose information restricts the application of SDVAC to mobile robots.

To overcome the two difficulties (i.e., formulating the stability criterion and estimating the human arm stiffness in the mobile robot-based pHRI), we developed a mobile robot which is able to measure the relative human position with respect to the robot using four on-board LRFs. Based on the developed robotic system, the following methods are proposed:

- The robot is controlled so as to have admittance-type dynamics in the human frame. We show that the human frame admittance control can avoid the nonlinearity in the system dynamics and that the stability criterion can be formulated using linear system techniques.

- With the human position information, the human arm stiffness can be estimated. Based on an improved stiffness estimator, SDVAC is implemented on the mobile robot.

With the above methods, we can stabilize the mobile robotbased pHRI and enhance its performance by adjusting the admittance parameters online. The proposed methods, as well as the developed robotic system, have the potential to be applied to human worker assistance, rehabilitation and robot dance partners [14 - 17].

This paper is organized as follows: Section 2 introduces the robotic system. The admittance control in the human-frame is discussed in Section 3. Section 4 formulates the stability criterion for the human-frame admittance control. Section 5 presents the improved stiffness estimator and the designed controller. Section 6 presents and discusses the results of simulations and experiments. Conclusions are presented in Section 8.

\section{System Description}

The developed robotic system (shown in Figure 1) is a test bed for a dance partner and walking support robots. This mobile robot has four omni-directional wheels, each

1 In some of the literature [19], admittance control may also be referred to as "impedance control". However, in this paper we follow the naming conventions of "admittance" and "impedance" made in [18]. 
actuated by a 250W DC servo motor. The maximum moving speed of the robot is $1.3 \mathrm{~m} / \mathrm{s}$, which is approximately equal to the normal walking speed of a human. The control algorithm is implemented in real-time on an onboard PC with the QNX operating system.

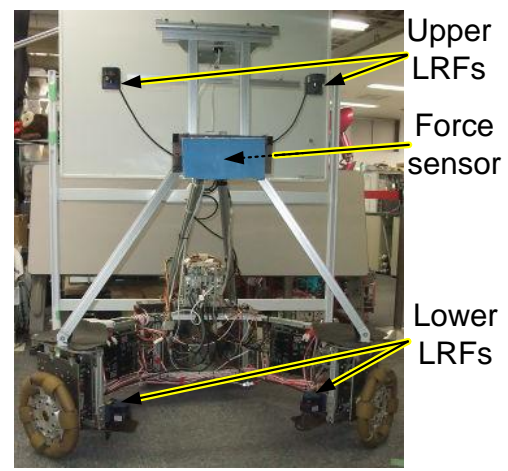

Figure 1. The developed robotic system - the force/torque sensor is installed in the waist of the robot (behind the blue plate)

Besides the encoders (installed on the motor shafts) and the force/torque sensor (installed in the waist of the robot) necessary for the admittance control, the mobile robot is also equipped with four LRFs (Hokuyo UBG-04LX-F01), with two upper and two lower LRFs monitoring the chest and each ankle of the human user respectively. In our earlier work, we implemented two LRFs to measure both the chest and the ankles (see Figure 2).

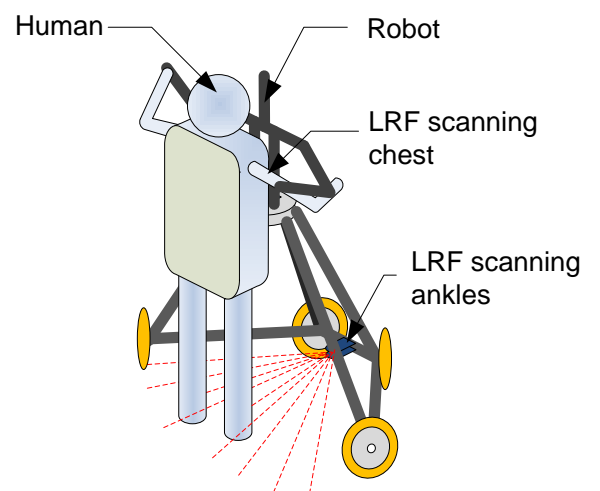

Figure 2. In our earlier work, the chest and ankle positions of the human were measured using individual LRFs

However, in the current robotic system shown in Figure 1, we use two pairs of LRFs to improve the quality of the measurements:

- For measuring the chest, the paired LRFs have significantly greater coverage than a single LRF (see Figure 3).

- A single LRF can measure both ankles when the human user is performing simple tasks (e.g., maintaining a static posture, as in Figure 2). However, when the tasks are complicated (e.g., when the human is rotating around the vertical axis or when the second through to the fifth sub-tasks in Figure 13 are being performed), the crossing of the ankles may occur (as shown in Figure 4), leading to occlusion problems (see Figure 5(a)). In this case, the positions of the ankles cannot be measured by a single LRF. Therefore, we implement a pair of LRFs to overcome this occlusion problem, as shown in Figure 5(b).

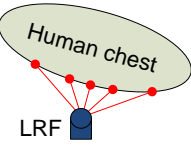

(a) Single LRF

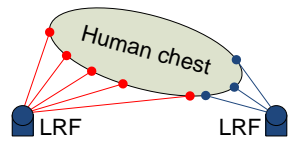

(b) Paired LRFs
Figure 3. When measuring the chest of the human user, the paired LRFs (b) have significantly greater coverage than a single LRF (a)

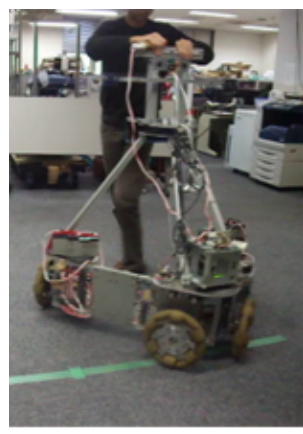

(a) Ankle occlusion

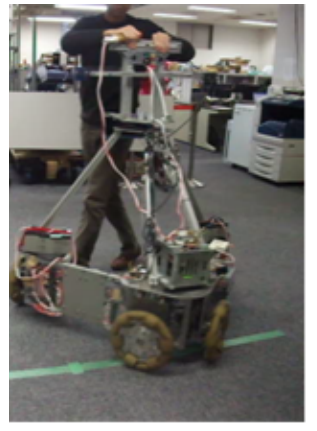

(b) Ankle crossing
Figure 4. Ankle occlusions may occur when performing relatively complicated tasks

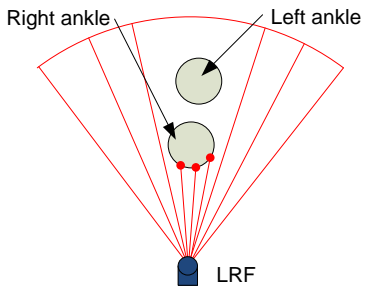

(a) Single LRF

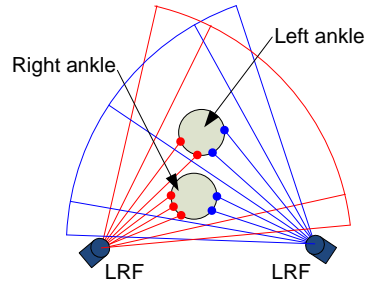

(b) Paired LRFs
Figure 5. When measuring the ankles of the human, the use of paired LRFs can eliminate occlusion, as illustrated in (a)

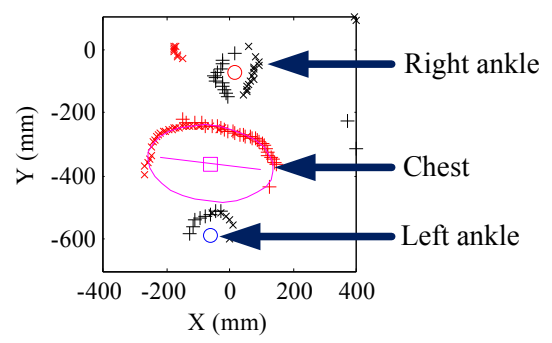

Figure 6. A range image of the chest and the ankles. The black " + " and " $x$ " markers denote the raw range data from the two lower LRFs. The red " + " and " $x$ " markers stand for the range data from the two upper LRFs. Both " $\mathrm{O}$ " and " $\square$ " mark the calculated positions of the ankles and the chest respectively. 


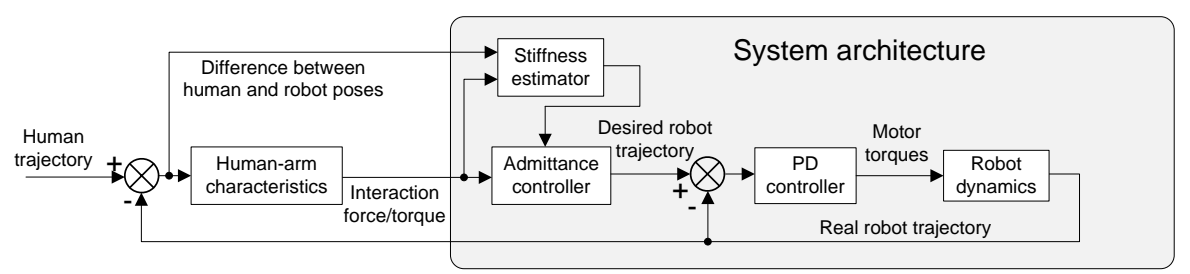

Figure 7. Block diagram of the proposed system based on SDVAC

With the two pairs of LRFs, we can obtain range images of the chest and the ankles (shown in Figure 6). Using the range data, the positions of the chest and the ankles are calculated following the steps listed below:

1. The chest and the ankles appearing in the range image are considered as an ellipse and two circles, respectively. At the beginning of each pHRI, the human user is required to stand near the robot for a few seconds, until the geometric parameters (i.e., the lengths of the major and minor axes of the ellipse, and the radius of the circles) are extracted.

2. Given a specific range image obtained during the pHRI, we first remove the spurious range image data that are significantly detached from the chest/ankle patterns.

3. Using the ellipse and circle parameters obtained in Step 1, the chest centre and its orientation are calculated by ellipse fitting while the ankle centres are obtained by circle fittings.

The calculated chest/ankle positions in a range image are shown in Figure 6. Using the calculated human-chest position, a stiffness estimator is introduced (Figure 7). The estimated stiffness is used to adjust the parameters of the admittance controller, whose output (the desired robot trajectory) is tracked by a low-level PD controller. The block diagram of the SDVAC-based system is given in Figure 7.

Besides estimating stiffness, the measured human position is also utilized to determine the human coordinate frame in which we can define the admittance-type dynamics of the mobile robot. In the next section, we discuss the implementation of admittance controllers in different coordinate frames.

\section{Admittance Control in Different Coordinate Frames}

An admittance controller needs to be defined in a coordinate frame. In our application, where a human user physically interacts with a mobile robot on the ground, it is natural to consider three candidate frames: the global frame $\Sigma_{g}$ (fixed to the ground), the robot frame $\Sigma_{r}$ and the human frame $\Sigma_{h}$. Figure 8 shows a top-view of the three frames. For ease of modelling and without loss of generality, the origin of the human frame is defined as a point in front of the human user so that the robot is at the desired pose when $\Sigma_{r}$ and $\Sigma_{h}$ are aligned.

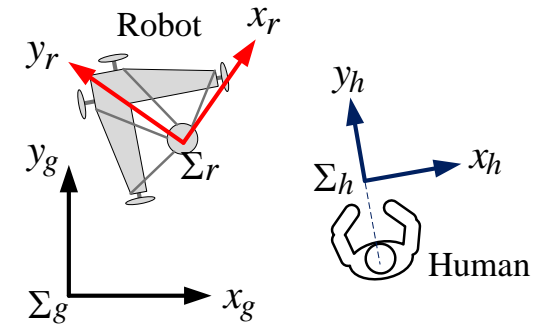

Figure 8. Top-view showing the ground, the robot and the human frames

Both the human and the robot are assumed to have three degrees of freedom (DOF). These are two-DOF translational motion along the horizontal ground and one-DOF rotation around the vertical axis. For convenience, we name them as the $x / y$-dimensions and the $\theta$-dimension, respectively.

The admittance control law of the robot is given by

$$
\ddot{p}=M^{-1}(f-C \dot{p}),
$$

where $p=[x, y, \theta]^{T}$ is the robot pose. $M$ and $C$ are $\mathbb{R}^{3 \times 3}$ virtual mass and viscosity matrices of the admittance controller, respectively. $f=\left[f_{x}, f_{y}, f_{\theta}\right]^{T}$ is the force/torque vector acting on the robot.

The admittance-type dynamics defined in (1) do not correspond to any coordinate frame. In the next subsection, we show how different selections of coordinate frames can lead to different admittance-type dynamics in pHRI.

\subsection{Admittance Control in the Global Frame}

The admittance control law in the global frame is

$$
\ddot{p}_{r}^{g}=M_{g}^{-1}\left(f_{r}^{g}-C_{g} \dot{p}_{r}^{g}\right)
$$

where $\boldsymbol{p}_{r}^{g}$ is the robot pose in the global frame (the subscript " $r$ " indicates the robot and the superscript " $g$ " indicates the global frame). $f_{r}^{g}$ is the force/torque vector acting on the robot. $\boldsymbol{M}_{g}$ and $\boldsymbol{C}_{g}$ are the virtual mass and viscosity matrices. 
If the human and the robot are separated, (2) is a simple linear system. However, nonlinearity arises when the human and the robot are physically coupled via the human's arms. For simplicity, we approximate this arm connection with a stiffness matrix and ignore the effect of viscosity ${ }^{2}$. With the stiffness matrix, the interaction force is modelled by

$$
f_{r}^{r}=\boldsymbol{K}_{r} \boldsymbol{p}_{h}^{r}
$$

where $f_{r}^{r}, p_{h}^{r}$ and $K_{r}$ are the force/torque, the human pose and the stiffness matrix defined in the robot frame, respectively. Theoretically, $\boldsymbol{K}_{r}$ can be defined in any frames, but it is more convenient to define $K_{r}$ in the robot or human frame, and thus $\boldsymbol{K}_{r}$ is only affected by the human arm characteristics. In contrast, if $\boldsymbol{K}_{r}$ is defined in the global frame, then $\boldsymbol{K}_{r}$ will depend on the orientation of the robot. This orientation-dependency can significantly complicate the system and is explained in (6).

The human pose is transformed to the global frame by

$$
\boldsymbol{p}_{h}^{g}=\boldsymbol{R}_{r}^{g} \boldsymbol{p}_{h}^{r}+\boldsymbol{p}_{r}^{g}
$$

where $\boldsymbol{p}_{r}^{g}$ is the robot pose in the global frame, and $\boldsymbol{R}_{r}^{g}$ is a $\mathbb{R}^{3 \times 3}$ rotation matrix (the rotation angle $\theta_{r}^{g}$ is the third component of $\boldsymbol{p}_{r}^{g}$ ) from the robot frame to the global frame. $\boldsymbol{R}_{r}^{g}$ has its third row as $[0,0,1]$ and the third column as $[0,0,1]^{T}$.

Similarly, the transformation from $f_{r}^{r}$ to $f_{r}^{g}$ is

$$
f_{r}^{g}=\boldsymbol{R}_{r}^{g} f_{r}^{r}
$$

From (2), (3), (4) and (5), the system dynamics in the global frame are

$$
\begin{aligned}
{\left[\begin{array}{c}
\dot{\boldsymbol{p}}_{r}^{g} \\
\ddot{\boldsymbol{p}}_{r}^{g}
\end{array}\right] } & =\left[\begin{array}{cc}
\mathbf{0}_{3 \times 3} & \boldsymbol{I}_{3 \times 3} \\
-\boldsymbol{M}_{g}^{-1} \boldsymbol{K}\left(\theta_{r}^{g}\right) & -\boldsymbol{M}_{g}^{-1} \boldsymbol{C}_{g}
\end{array}\right]\left[\begin{array}{c}
\boldsymbol{p}_{r}^{g} \\
\dot{\boldsymbol{p}}_{r}^{g}
\end{array}\right] \\
& +\left[\begin{array}{c}
\mathbf{0}_{3 \times 3} \\
\boldsymbol{M}_{g}^{-1} \boldsymbol{K}\left(\theta_{r}^{g}\right)
\end{array}\right] \boldsymbol{p}_{h}^{g},
\end{aligned}
$$

where $I_{3 \times 3}$ is a three-by-three identity matrix. $\boldsymbol{K}\left(\theta_{r}^{g}\right)=\boldsymbol{R}_{r}^{g} \boldsymbol{K}_{r} \boldsymbol{R}_{g}^{r}$ is a $\theta_{r}^{g}$-dependent stiffness matrix which introduces nonlinearity into (6).
Due to the nonlinearity, the stability of (6) cannot be examined by the Routh-Hurwitz criterion (as in [35]). Instead, a Lyapunov function (as in [38]) is formulated as a sufficient condition for stability. Here, we introduce a Lyapunov function using the mechanical energy of the human/robot system:

$$
V\left(\boldsymbol{p}_{r}^{g}, \dot{\boldsymbol{p}}_{r}^{g}\right)=\frac{1}{2}\left(\boldsymbol{p}_{r}^{g}\right)^{T} \boldsymbol{K}\left(\theta_{r}^{g}\right) \boldsymbol{p}_{r}^{g}+\frac{1}{2}\left(\dot{\boldsymbol{p}}_{r}^{g}\right)^{T} \boldsymbol{M}_{g} \dot{\boldsymbol{p}}_{r}^{g}
$$

We directly give the result of $\dot{V}=\mathrm{d} V / \mathrm{d} t$. Assuming an autonomous system with the input $\boldsymbol{p}_{h}^{g}=\mathbf{0}$, and given that $\boldsymbol{K}_{r}, \boldsymbol{M}_{g}$ and $\boldsymbol{C}_{g}$ are all symmetric, we have

$$
\dot{V}=-\left(\dot{\boldsymbol{p}}_{r}^{g}\right)^{T} \boldsymbol{C}_{g} \dot{\boldsymbol{p}}_{r}^{g}+\dot{\theta}_{r}^{g}\left(\boldsymbol{p}_{r}^{g}\right)^{T} \hat{\boldsymbol{S}} \boldsymbol{p}_{r}^{g}
$$

where $\hat{\boldsymbol{S}}=\hat{\boldsymbol{R}}_{r}^{g} \boldsymbol{K}_{r} \boldsymbol{R}_{g}^{r}+\boldsymbol{R}_{r}^{g} \boldsymbol{K}_{r} \hat{\boldsymbol{R}}_{g}^{r}$ and $\hat{\boldsymbol{R}}_{r}^{g}=\mathrm{d}\left(\boldsymbol{R}_{r}^{g}\right) / \mathrm{d} \theta_{r}^{g}$. If $\boldsymbol{K}_{r}$ is symmetric, $\hat{S}$ is thus skew-symmetric; hence, $\theta_{r}^{g}\left(\boldsymbol{p}_{r}^{g}\right)^{T} \hat{\boldsymbol{S}} \boldsymbol{p}_{r}^{g}=0$ and $\dot{V}=-\left(\dot{\boldsymbol{p}}_{r}^{g}\right)^{T} \boldsymbol{C}_{g} \dot{\boldsymbol{p}}_{r}^{g}$. Therefore, the system is stable given that $\boldsymbol{K}_{r}$ and $\boldsymbol{M}_{g}$ are symmetric and $\boldsymbol{C}_{g}$ is positive definite.

However, although the global-frame admittance control is stable, it exposes two problems in (6), (7), (8) and (2):

- The first problem is nonlinearity, which is introduced by transforming $K_{r}$ to the global frame, resulting in an orientation-dependent stiffness matrix $K\left(\theta_{r}^{g}\right)$. Although the stability of the nonlinear system (6) has been proved, if extra dynamics (e.g., a low-pass filter or a PD controller) are introduced, formulating the Lyapunov function will become much more challenging.

- The second problem lies in (2), where $M_{g}$ and $C_{g}$ are defined in the global frame. Suppose that the human and the robot are rotating together while maintaining a constant relative pose, then the human user may feel different admittances depending on $\theta_{r}^{g}$. This orientation dependency leads to undesired user experiences in $\mathrm{pHRI}^{3}$

\subsection{Admittance Control in the the Robot Frame}

The admittance control in the robot frame is

$$
\ddot{\boldsymbol{p}}_{r}^{r}=\boldsymbol{M}_{r}^{-1}\left(f_{r}^{r}-C_{r} \dot{\boldsymbol{p}}_{r}^{r}\right)
$$

2 On the one hand, the proposed method can also handle the inclusion of arm viscosity, and hence generality is preserved. On the other hand, the viscosityfree assumption usually leads to conservative controller designs [26], and hence stability is also preserved.

3 One possible solution to this problem is to adjust $M_{g}$ and $C_{g}$ according to $\theta_{r}^{g}$, and thus the human user can feel the same admittance for all $\theta_{r}^{g}$. However, this method needs the measurement of $\theta_{r}^{g}$. Since odometry is prone to accumulative errors, it is necessary to refer to some external information to determine the orientation of the robot in the global frame. However, the need for external information introduces additional restrictions on the pHRI. For example, $\theta_{r}^{g}$ could be measured by a motion capture system, an on-board camera, or a magnetometer; however, they would require the robot to be used in a motion capture room, in an environment with some visual features or in a non-distorted magnetic field. 
where $\dot{\boldsymbol{p}}_{r}^{r}$ and $\ddot{\boldsymbol{p}}_{r}^{r}$ are the robot velocity and acceleration in the robot frame at a specific instant.

By taking the time-derivative of (4), and using the facts that $\dot{\theta}_{r}^{g}=\dot{\theta}_{r}^{r}$ and $\dot{\boldsymbol{p}}_{r}^{g}=\boldsymbol{R}_{r}^{g} \dot{\boldsymbol{p}}_{r}^{r}$, we have

$$
\dot{\boldsymbol{p}}_{h}^{r}=-\dot{\theta}_{r}^{r} \hat{\boldsymbol{H}} \boldsymbol{p}_{h}^{r}-\dot{\boldsymbol{p}}_{r}^{r}+\boldsymbol{R}_{g}^{r} \dot{\boldsymbol{p}}_{h}^{g},
$$

where $\hat{\boldsymbol{H}}$ is a skew-symmetric matrix with $\hat{\boldsymbol{H}}_{(1,2)}$ (the subscripts denote the row and column indices) being -1 , $\hat{H}_{(2,1)}=1$, and all the other entries in $\hat{H}$ are 0 .

From (9), (10) and (3), the system dynamics are given by

$$
\begin{aligned}
{\left[\begin{array}{c}
\ddot{\boldsymbol{p}}_{r}^{r} \\
\dot{\boldsymbol{p}}_{h}^{r}
\end{array}\right] } & =\left[\begin{array}{cc}
-\boldsymbol{M}_{r}^{-1} \boldsymbol{C}_{r} & \boldsymbol{M}_{r}^{-1} \boldsymbol{K}_{r} \\
-\boldsymbol{I}_{3 \times 3} & -\dot{\theta}_{r}^{r} \hat{\boldsymbol{H}}
\end{array}\right]\left[\begin{array}{c}
\dot{\boldsymbol{p}}_{r}^{r} \\
\boldsymbol{p}_{h}^{r}
\end{array}\right] \\
& +\left[\begin{array}{c}
\boldsymbol{0}_{3 \times 3} \\
\boldsymbol{R}_{g}^{r}
\end{array}\right] \dot{\boldsymbol{p}}_{h}^{g} .
\end{aligned}
$$

Compared with the global frame admittance control in (6), the robot frame admittance control in (11) does not have the orientation-dependency problem. In addition, (11) contains only one nonlinear component $\left(\dot{\theta}_{r}^{r}\right)$ and looks simpler than (6). However, formulating a Lyapunov function for (11) is actually much more challenging (e.g., the dissipative-energy condition used in (8) is not preserved here). The authors are still trying to formulate a Lyapunov function for (11). Moreover, we might also expect that, if extra filter/controller dynamics are introduced, the stability analysis will become even more non-trivial.

\subsection{Admittance Control in the Human Frame}

The admittance control in the human frame is

$$
\ddot{\boldsymbol{p}}_{r}^{h}=\boldsymbol{M}_{h}^{-1}\left(f_{r}^{h}-C_{h} \dot{\boldsymbol{p}}_{r}^{h}\right)
$$

The force/torque defined in the human frame is

$$
f_{r}^{h}=-K_{h} p_{r}^{h}
$$

With (12) and (13), the system dynamics in the human frame are

$$
\left[\begin{array}{c}
\dot{\boldsymbol{p}}_{r}^{h} \\
\ddot{\boldsymbol{p}}_{r}^{h}
\end{array}\right]=\left[\begin{array}{cc}
\mathbf{0}_{3 \times 3} & \boldsymbol{I}_{3 \times 3} \\
-\boldsymbol{M}_{h}^{-1} \boldsymbol{K}_{h} & -\boldsymbol{M}_{h}^{-1} \boldsymbol{C}_{h}
\end{array}\right]\left[\begin{array}{c}
\boldsymbol{p}_{r}^{h} \\
\dot{\boldsymbol{p}}_{r}^{h}
\end{array}\right] .
$$

Since (14) is linear, its stability can be tested with the RouthHurwitz criterion. Instead of directly checking the $\mathbb{R}^{6 \times 6}$ state matrix, we use the determinant property of block matrices, leading to the following characteristic equation,

$$
\operatorname{det}\left(\lambda^{2} \boldsymbol{I}+\lambda \boldsymbol{M}_{h}^{-1} \boldsymbol{C}_{h}+\boldsymbol{M}_{h}^{-1} \boldsymbol{K}_{h}\right)=0
$$

where $\lambda$ is the eigenvalue of the state matrix and $\operatorname{det}(\cdot)$ denotes the determinant of a given matrix.

Besides the Routh-Hurwitz test, we can also use a similar Lyapunov function as in (7). By assuming that $\boldsymbol{K}_{h}, \boldsymbol{M}_{h}$ and $\boldsymbol{C}_{h}$ are positive-definite, we obtain $\dot{V}=-\left(\dot{\boldsymbol{p}}_{r}^{h}\right)^{T} \boldsymbol{C}_{h} \dot{\boldsymbol{p}}_{r}^{h}$ and the system stability is proved.

Besides the linear dynamics, another use of the human frame admittance control is to decouple the three-DOF robot dynamics in the human frame. If we select the virtual mass $\boldsymbol{M}_{h}$ and the virtual viscosity $\boldsymbol{C}_{h}$ as diagonal and we assume that the stiffness $K_{h}$ can be approximated by a diagonal matrix ${ }^{4}$ as in [19], then the dynamics in (14) can be decoupled. This decoupling largely reduces the system complexity and enables us to obtain a stability criterion. In the next section, we derive the stability criterion for the human frame admittance control.

\section{Stability of the Human Frame Admittance Control}

The dynamics in the three dimensions can be decoupled; therefore, without loss of generality, we consider the robot dynamics in the $x$-dimension,

$$
\ddot{x}=m_{x}^{-1}\left(-k_{x} x-c_{x} \dot{x}\right),
$$

where $k_{x}, m_{x}$ and $c_{x}$ are the first diagonal entries in $\boldsymbol{K}_{h}, \boldsymbol{M}_{h}$ and $C_{h}$, respectively. The above system is stable if $k_{x}, m_{x}$ and $c_{x}$ are all positive.

However, although the admittance controller itself is stable, when it is combined with other components, instabilities may arise. For example, Duchaine studied the effect of a low-pass-filter [26] in a haptic device with slight inertia and friction. In contrast, our system is a human-size mobile robot which has significant inertia and is subject to significant ground friction. Therefore, a PD controller is used for reference trajectory tracking. Besides the PD

4 In the diagonal matrix approximation of $\boldsymbol{K}_{h}$, as the principal axes of the ellipsoid-shaped stiffness is restricted so as to be aligned with the axes of the defined coordinate frame, the accuracy of the stiffness estimations is reduced [27]. In this paper, to simplify the stability analysis, we use the assumption in [19], while the consequence of the diagonal matrix approximation on the pHRI stability is to be examined in experiments. 
controller, another factor in our system is the relatively long sampling period (28ms) of the LRF used for sensing the human. This discretization also influences stability. Therefore, in this section, we study the stability criterion which takes the discretization and the PD controller into account.

At moment $k$, let the robot state be $x(k)=[x(k), \dot{x}(k)]^{T}$. The admittance controller generates a desired acceleration $\ddot{x}_{d}$ using (16). The desired robot state at moment $k+1$ is:

$$
x_{d}(k+1)=\left[\begin{array}{cc}
1 & t_{s} \\
0 & 1
\end{array}\right] x(k)+\left[\begin{array}{c}
t_{s}^{2} \\
t_{s}
\end{array}\right] \ddot{x}_{d}
$$

where $t_{s}$ is the sampling period. With the current state $x(k)$ and the desired state $x_{d}(k+1)$, the PD controller generates an actuation force:

$$
\tau_{x}=\left[\begin{array}{ll}
k_{p} & k_{D}
\end{array}\right]\left(x_{d}(k+1)-x(k)\right)+k_{A} \ddot{x}_{d},
$$

where $k_{P}, k_{D}$ and $k_{A}$ are controller gains. Due to the discretization, the actuation force $\tau_{x}$ is held (by the $\mathrm{ZOH}$, i.e., zeroorder hold) until moment $k+1$ and the robot is driven to a new state $x(k+1)$. We approximate the mechanical admittance of the robot with a first-order system:

$$
\begin{aligned}
& \ddot{\boldsymbol{x}}=\boldsymbol{A}_{c} \boldsymbol{x}+\boldsymbol{B}_{c} \tau_{x} \\
& \boldsymbol{A}_{c}=\left[\begin{array}{cc}
0 & 1 \\
0 & -m_{R}^{-1} d_{R}
\end{array}\right] \\
& \boldsymbol{B}_{c}=\left[0,-m_{R}^{-1}\right]^{T},
\end{aligned}
$$

where $m_{R}$ and $d_{R}$ are the physical mass and viscosity, respectively. The discretized system dynamics are

$$
x(k+1)=\left(A_{d}+B_{d} L\right) x(k),
$$

where $A_{d}$ and $B_{d}$ are the discretized forms of $A_{c}$ and $B_{c^{\prime}}$ respectively. $L$ is a $\mathbb{R}^{1 \times 2}$ row vector with $L_{(1,1)}=m_{x}^{-1} k_{x}\left(-k_{P} t_{s}^{2}-k_{D} t_{s}-k_{A}\right)$ and $L_{(1,2)}=m_{x}^{-1} c_{x}\left(-k_{P} t_{s}^{2}-k_{D} t_{s}-k_{A}\right)+k_{P} t_{s}$.

System (20) is stable if the two eigenvalues of $A_{d}+B_{d} L$ are both inside the unit circle. Since $A_{d}$ and $\boldsymbol{B}_{d}$ are assumed to be given by identification, while $k_{P}, k_{D}, k_{A}$ and $t_{s}$ are all known, we can derive an analytic stability condition using the Jury stability test. For example, if $m_{R}=k_{A}=70, d_{R}=40$, $k_{P}=1600, k_{D}=880$ and $t_{s}=0.028$, the stability criterion is given by $c_{x}>0.05 m_{x}+0.01 k_{x}$.

\section{Stiffness-based Variable Admittance Control}

\subsection{Stiffness Estimation}

In the previous section, we showed that, to guarantee the stability of pHRI, the admittance parameters should satisfy a stiffness-dependent inequality. Therefore, identifying arm stiffness is the prerequisite for variable admittance control [19].

In previous investigations, arm stiffness has been estimated by performing a linear regression on a fixed number of samples of the force/position measurements [19]. However, if the human arm stiffness rapidly changes (which frequently occurs in pHRI), fitting the fixed-size samples may temporarily generate poor results. An example is given in Figure 9. Assume that the number of force/position samples used in the linear regression is 20. At moment $k$, the samples are $x_{k-19}, x_{k-18}, \ldots$ and $x_{k}$. We also assume that the arm stiffness rapidly increases between the sampling moments of $x_{k-5}$ and $x_{k-4}$. The 20 samples are shown in Figure 9(a). Because of the rapid stiffness change, the 20 samples diverge into two groups. Consequently, performing a linear regression on all 20 samples leads to an incorrect fitting result, which is shown in Figure 9(a).

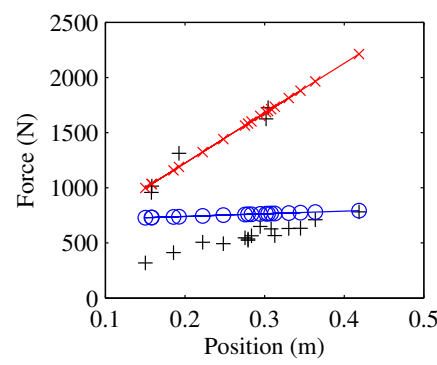

(a) Comparing fitting methods

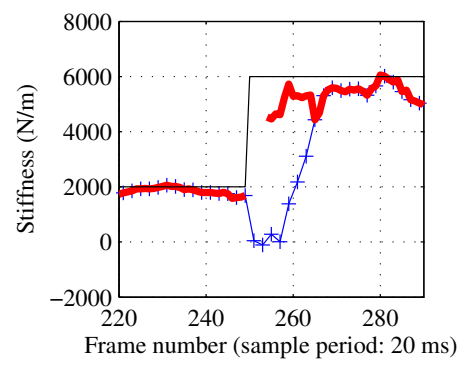

(b) Comparing estimation results

Figure 9. When the stiffness changes rapidly, the proposed method gives a better fitting result. In (a), the " + " markers denote the sampled data. Segments with the circular and crossed markers show the fitting results of the original method and the proposed method respectively. In (b), the thin, plain curve is the true stiffness. The " + " markers and the thick curves show the results of the original method and the proposed method respectively.

The poor fitting result, which is caused by rapid stiffness changes, can be solved by using samples of a variable size: Instead of fitting a fixed number of samples, we can perform linear regressions on multiple possible sample sizes until the optimal fit is found. To determine which 
fitting result is optimal, we can use the correlation coefficients as the index of optimality. The proposed estimation method is given in Algorithm 1. Results from the original and the proposed stiffness-estimation methods are compared in Figure 9(b). According to the comparison, we can see that the proposed method performs better under a rapid stiffness change.

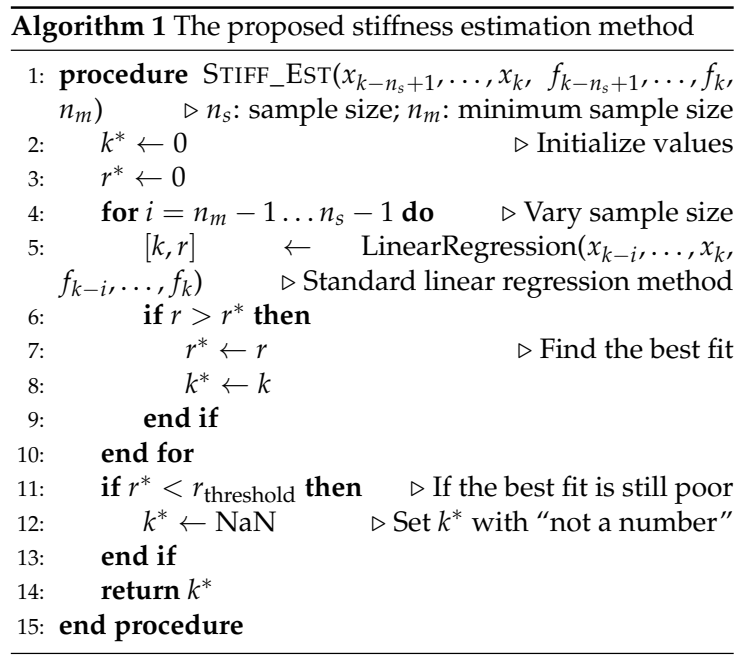

\subsection{The Stiffness-based Variable Admittance Control}

Theoretically, both the virtual mass $m_{x}$ and the virtual viscosity $c_{x}$ can be varied in SDVAC. However, in practice, the virtual mass $m_{x}$ is usually restricted by other factors. For example, in our system, the force sensor is installed in the waist of the robot and there is an upper-body structure above the force sensor (see Figure 1). Due to the inertia of the upper body, $m_{x}$ cannot be arbitrarily small. Otherwise, the small $m_{x}$ may lead to large robot accelerations and the upper body will conversely apply significant inertial forces on the force sensor, introducing noise or even instabilities. Because of this constraint, in this paper, we keep the virtual mass constant and only vary the virtual viscosity.

The system parameters are listed in Table 1, with the sampling period $t_{s}=28 \mathrm{~ms}$. The relationships between the virtual mass and viscosity are shown in Figure 10.

\begin{tabular}{ccccccc}
\hline Dimensions & $m_{R}$ & $d_{R}$ & $k_{P}$ & $k_{D}$ & $k_{A}$ & $m$ \\
\hline$x$ & 56 & 112 & 698 & 384 & 0.34 & 15 \\
\hline$y$ & 56 & 112 & 698 & 384 & 0.34 & 15 \\
\hline$\theta$ & 15 & 42 & 349 & 192 & 0.19 & 1 \\
\hline
\end{tabular}

Table 1. The parameters used in the SDVAC design and simulations. $m_{R}$ and $d_{R}$ were identified in a preliminary test using the step response of the robot to motor torque inputs.

The values in the contour denote max $\|\lambda\|^{2}$ of the matrix $A_{d}+B_{d} L$ in (20), where values greater than 1 indicate instability. According to Figure 10, for a given contour line, the stiffness and virtual viscosity has a linear relationship. This conforms to the result of Section 4.

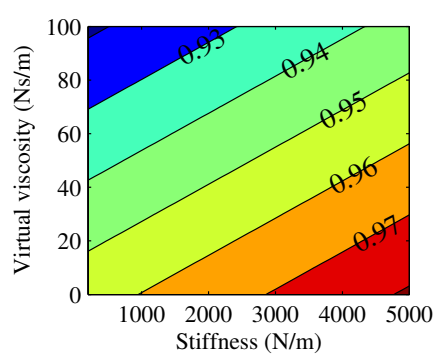

(a) $x$ and $y$-dimensions

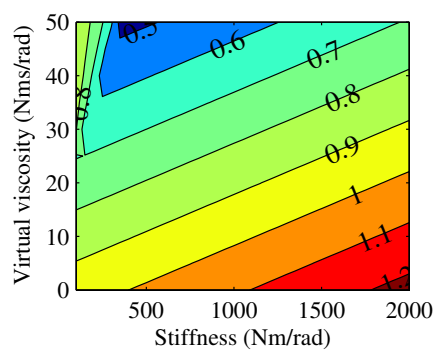

(b) $\theta$-dimension

Figure 10. Contours of the pHRI stability with respect to human arm stiffness and virtual viscosity. The contour values greater than 1 indicate instability.

Therefore, we choose the following linear rules for SDVAC:

$$
\begin{aligned}
& c_{x}=60 k_{x} / 4500+10 \\
& c_{y}=60 k_{y} / 4500+10 \\
& c_{\theta}=25 k_{\theta} / 1800+5,
\end{aligned}
$$

which is to be experimentally tested in Section 6.2.

\subsection{Compensating for the Human's Future Trajectories}

Besides SDVAC, the pHRI performance may also be improved by estimating and compensating for the human's future trajectories. The existing methods for future trajectory estimation in $[27,30,32]$ cannot be directly applied to our application, where the human user walks with the robot and frequently varies his/her direction of motion. Consequently, the force information is often a result of body dynamics rather than a sign of human intention. Therefore, in the following, we propose and test a new heuristic method.

Empirically, a human's future trajectory is correlated with his/her feet positions and velocities. Let $\boldsymbol{q}_{L}^{g}, \boldsymbol{q}_{S}^{g} \in \mathbb{R}^{2}$ be the positions of the landing and swinging feet in the global frame, respectively. $\dot{q}_{S}^{g}$ is the swinging foot velocity. Using the measured ankle information, we implement a heuristic compensation method which generates a virtual compensation force in two situations: 
1. The swinging foot has a non-zero velocity $\dot{\boldsymbol{q}}_{S}^{g}$, and

2. $\boldsymbol{q}_{L}^{g}-\boldsymbol{q}_{S}^{g}$ and $\dot{\boldsymbol{q}}_{S}^{g}$ form an acute angle.

The above rules give

$$
f_{V}^{g}=a_{1} h(\beta) \beta\left(\boldsymbol{q}_{L}^{g}-\boldsymbol{q}_{S}^{g}\right)+a_{2} \dot{\boldsymbol{q}}_{S}^{g}
$$

where $f_{V}^{g}$ is the virtual compensation force in the global frame. $a_{1}$ and $a_{2}$ are the user-specified gains (determined in preliminary trials according to the human user's subjective evaluations of the compensation intensity). $h(\cdot)$ is the unit step (or Heaviside) function, and $\beta$ is defined as

$$
\beta=\dot{\boldsymbol{q}}_{S}^{g} \cdot\left(\boldsymbol{q}_{L}^{g}-\boldsymbol{q}_{S}^{g}\right) /\left(\left\|\dot{\boldsymbol{q}}_{S}^{g}\right\|+\epsilon\right),
$$

where $\epsilon>0$ is a small value used for avoiding singularity. $f_{V}^{g}$ will later be added as a virtual compensation force to the input of the admittance controller. Note that the proposed compensation method does not work in the $\theta$ dimension, as the human's feet trajectory can hardly be used for predicting future rotations.

\section{Simulations and Experiments}

\subsection{Simulations}

In the first simulation, we assumed the human user is static with $p_{h}^{g}=[0.1,0.2,0.3]^{T}$. The stiffness matrix was $\boldsymbol{K}_{h}=\operatorname{diag}(4500,4500,1800)$. The responses of the robot using three admittance controllers were simulated with the parameters listed in Table 1 and the sampling period $t_{s}=28 \mathrm{~ms}$. Controller 1 had constant $C_{h}=\operatorname{diag}(70,70,30)$, Controller 2 had constant $C_{h}=\operatorname{diag}(10,10,5)$, and Controller 3 was SDVAC as in (21). The simulation results in the $\theta-$ dimension are given in Figure 11.

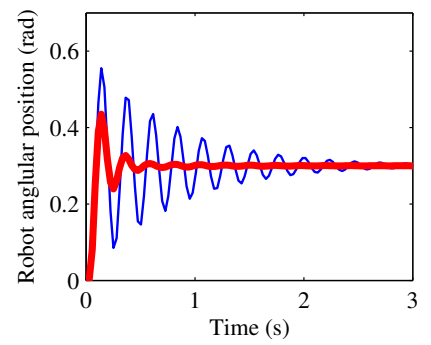

Figure 11. Responses of the robot in the $\theta$-dimension. The thin curve is the response of Controller 2 and the thick curves (overlapped) are the responses of Controllers 1 and 3.

As predicted by Figure 10(b), Controller 2 exhibited marginally stable behaviour. In contrast, Controller 3 (SDVAC) exhibited similar stable behaviour to Controller 1. Therefore, the use of SDVAC in stabilizing pHRI is supported and Controller 2 cannot be used under significant human arm stiffness.
In the second simulation, the orientation of the human was set to follow a sinusoidal trajectory with a period of $5 \mathrm{~s}$ and an amplitude of $\pi / 2$ (Figure 12(a)). The stiffness of the human arm was assumed to vary randomly between $0-$ $1800 \mathrm{~N} \mathrm{~m} / \mathrm{rad}$, and the standard deviation of the random changes was $100 \mathrm{~N} \mathrm{~m} / \mathrm{rad}$. Note that this random-walk stiffness was only used to qualitatively demonstrate the resultant pHRI of the admittance control (which is to be experimentally tested in the next subsection). The simulation results in the $\theta$-dimension are shown in Figure 12.

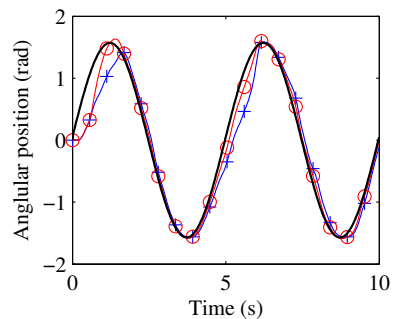

(a) Angular positions of human and robot

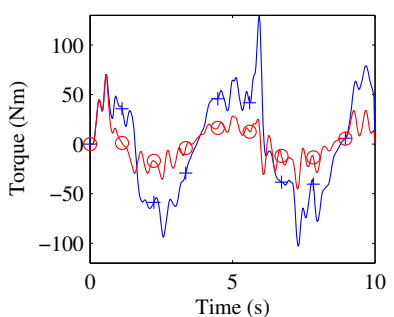

(b) Torque acting on robot
Figure 12. Simulation results in the $\theta$-dimension. In (a), the black curve represents the human trajectory, the blue curve with "+" markers denotes the robot trajectory using Controller 1 , and the red curve with " $\mathrm{o}$ " markers denotes the robot trajectory using Controller 3 . In (b), the blue curve with " + " markers is the interaction torque corresponding to Controller 1 while the red curve with " $\mathrm{o}$ " markers corresponds to controller 3.

We can see that the pHRI is stable and that the torque has been significantly reduced, suggesting the enhancement of pHRI performance by using SDVAC.

\subsection{Experiments}

In the experiments, we evaluated the pHRI performances of the following controllers:

1. A constant admittance controller (CAC) with $C_{h}=\operatorname{diag}(70,70,30)$;

2. SDVAC with $C_{h}$ varying according to (21);

3. (SDVAC-C) SDVAC with the compensation method given in (22).

The pHRI experiments were performed in an indoor environment with no obstacles in the experiment space. The robot used in the experiments was introduced in Section 2. To evaluate the performances of the three controllers, we designed a pHRI task containing the following steps:

1. Three markers were attached to the floor (see Figure 13). In Step 1, the human moves from the start marker to the end marker;

2. Turning $90^{\circ}$ in the clockwise direction;

3. Moving rightwards (seen from the human user) to the middle marker;

4. Moving leftwards to the end marker; 
5. Turning $90^{\circ}$ in the counter-clockwise direction;

6. Moving backwards to the start marker.

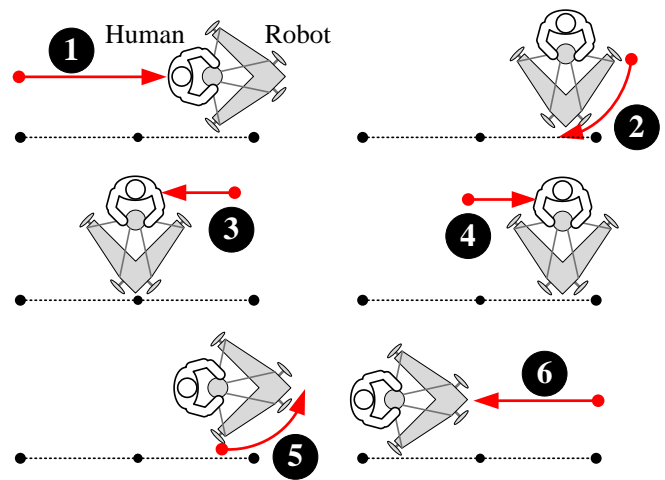

Figure 13. Procedure for the designed pHRI task - the three round dots at the bottom of each subfigure denote the start, middle and end markers attached to the floor. At each step, the human user is required to align the robot with a certain floor marker.

The above procedure is illustrated in Figure 13. A skilled user (who knew the operation of the mobile robot) and a novice user participated in the experiments. In the beginning, several preliminary trials were conducted to familiarize the novice user with the experimental procedure and the robot. For each user, the pHRI task was performed for three trials to compare the performance of CAC, SDVAC and SDVAC-C.

The experiment results are shown in Figure 14. Comparing these results in Table 2, we can observe that the force/torque interaction in the SDVAC and SDVAC-C trials are significantly smaller than the force/torque in the CAC trials. For example, in the $\theta$-dimension, the maximum torque has been reduced by more than $50 \%$ for both the skilled and the novice users. The significant force/torque reductions are also observed in the $x$ and $y$ dimensions. Since SDVAC and SDVAC-C demand less human effort in pHRI, their task completion times are also shorter: compared with CAC, both SDVAC and SDVAC-C correspond to over 32\% and $42 \%$ time reductions for the skilled and the novice users, respectively. Moreover, performances of the novice user are similar to the skilled user in the SDVAC and SDVAC$C$ trials, possibly because the simplicity of the admittance control demands little training on the human's part.

\begin{tabular}{lccc}
\hline & CAC & SDVAC & SDVAC-C \\
\hline $\begin{array}{l}\text { Max. force in the x- } \\
\text { dimension (N) }\end{array}$ & 38.3. (37.9) & 28.5(26.1) & 23.2(21.7) \\
\hline $\begin{array}{l}\text { Max. force in the y- } \\
\text { dimension (N) }\end{array}$ & $41.7(42.1)$ & $21.2(22.7)$ & $22.1(21.0)$ \\
\hline $\begin{array}{l}\text { Max. torque in the } \theta- \\
\text { dimension (Nm) }\end{array}$ & $17.7(17.7)$ & $7.0(6.8)$ & $6.4(6.7)$ \\
\hline $\begin{array}{l}\text { Task completion time (s) } \\
\text { 23.8(30.3) }\end{array}$ & $16.1(17.7)$ & $16.0(17.6)$ \\
\hline
\end{tabular}

Table 2. Comparison of the experimental results using the three listed controllers. The values inside and outside the parentheses denote the results of both the novice user and the skilled user, respectively.

We can also observe that SDVAC-C did not outperform SDVAC. Due to the complexity of human body dynamics during walking as well as the heuristic nature of SDVAC$\mathrm{C}$ in (22), it is a challenging task to quantitatively understand this phenomenon. However, empirically, the human users reported that SDVAC-C was "too active" and prone to "overshoot". An example of overshoot is the peak on the red curve at about 16s in Figure 14(e). This overshoot

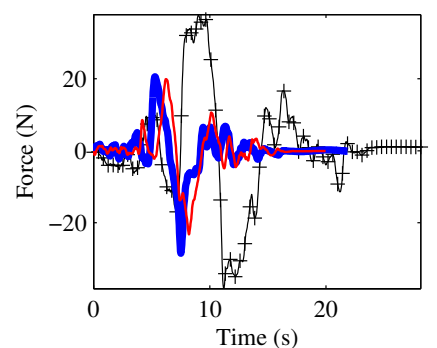

(a) Skilled user, $x$-dimension

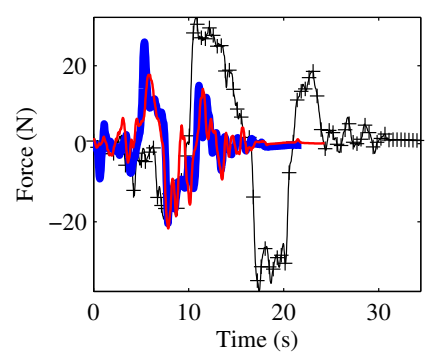

(d) Novice user, $x$-dimension

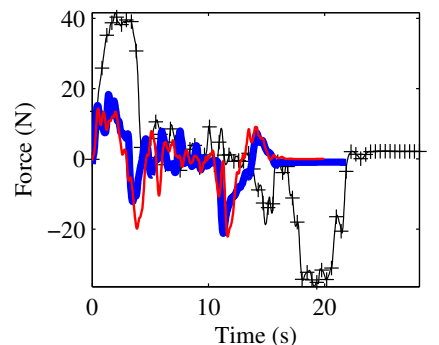

(b) Skilled user, $y$-dimension

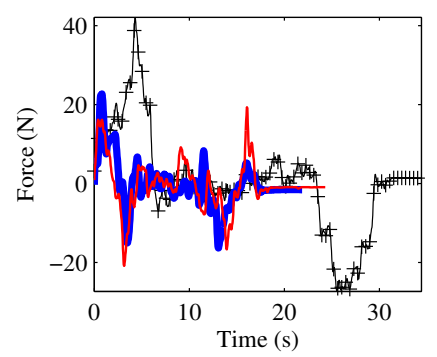

(e) Novice user, $y$-dimension

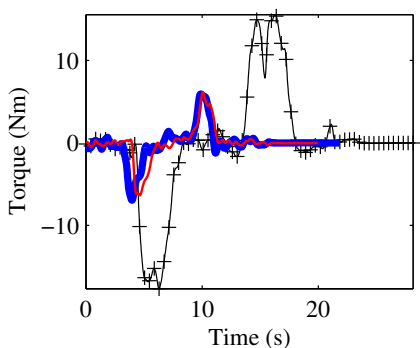

(c) Skilled user, $\theta$-dimension

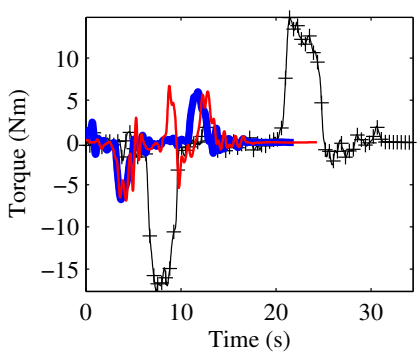

(f) Novice user, $\theta$-dimension

Figure 14. The results of pHRI performances using CAC, SDVAC and SDVAC-C. The thin black curves with the "+" markers denote the results of CAC. The thicker red curves stand for the results of SDVAC-C. The thickest blue curves correspond to the results of SDVAC. 
happened when the human user was making the transition from moving backwards to completely stopping (corresponding to the termination of step 6 in Figure 13). According to Figure 14(e), we can see that for CAC and SDVAC, the user spent roughly $5 \mathrm{~N}$ in order to decelerate the robot. In contrast, for SDVAC-C, to cancel the effect of ankle velocity compensation and realize the robot deceleration, it took the human user approximately $20 \mathrm{~N}$, which causes significant degeneration in pHRI performance. Consequently, as the human users had to resist any overshoots, the advantages gained from the compensations were cancelled.

According to the experimental results, and compared with CAC, the proposed SDVAC method significantly reduced the interaction force and task completion time in the mobile robot-based pHRI. Moreover, the performance of SDVAC$\mathrm{C}$ is similar to SDVAC.

\section{Discussion}

\subsection{Practicability of Robot Frame Admittance Control}

In practice, the robot frame admittance control implemented in the physical interaction between a human and a mobile robot demonstrates stable behaviours. However, due to its nonlinear dynamics in (11), its resultant pHRI stability is difficult to prove or disprove ${ }^{5}$. This implies that designing a variable-admittance rule for such a nonlinear system is of significant difficulty. Therefore, despite its practical stability, the robot frame admittance controller is not analysed or tested in the simulations or experiments.

\subsection{Possible Improvements on the Experiments}

The experiments discussed in Section 6.2 involved two subjects, and the use of SDVAC would receive stronger support if more subjects could be invited. At the same time, the fact that a low viscosity corresponds to a slight interaction force has been well-supported and applied in existing literature $[19,25,27,30-33,37]$. The viscosity of CAC is conservatively designed for the worst-case stiffness, whereas the viscosity of SDVAC is always smaller than or equal to (in the worst case) the viscosity of CAC. Therefore, the interaction force corresponding to SDVAC will be smaller than that of CAC. Figure 15 shows the viscosities of CAC and SDVAC. We can observe that the SDVAC viscosity is much smaller than the CAC viscosity for most of the experiment. Consequently, compared with the pHRI using CAC, the interaction force corresponding to SDVAC is significantly reduced. This observation matches the pHRI result of implementing SDVAC in human/manipulator interaction in [27, 37].

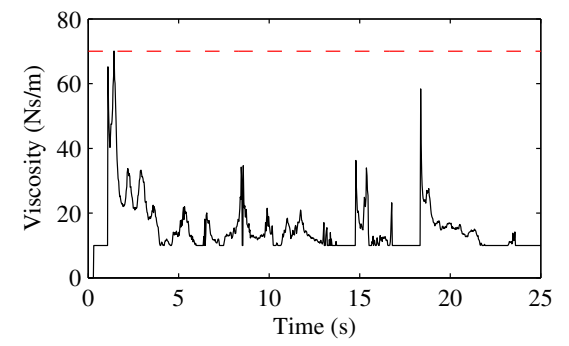

Figure 15. The viscosity parameters corresponding to the pHRI in 14(b). The dashed and solid curves denote the viscosities of CAC and SDVAC, respectively.

\subsection{The Implementation of LRFs in $p H R I$}

The reason for implementing LRFs instead of other sensors is the short distance $(0.06 \mathrm{~m}-0.6 \mathrm{~m})$ between the human and the robot. This short distance prevents the implementation of more commonly-used sensors (e.g., Kinect) in the pHRI. Moreover, as has been discussed in Section 2, in order to enlarge the coverage of human chest measurement and to prevent ankle occlusions (which frequently occur during complicated tasks), we implement four LRFs in total. In contrast, we performed tests using only two LRFs for detecting the upper body and the ankles in our earlier work $[40,41]$; however, it turned out that the detection was only successful for simpler tasks, e.g., when the human/robot motions were limited in the sagittal plane [40,41].

Because LRFs are expensive (each LRF costs approximately 1700 USD), the four LRFs implemented in the robot significantly elevate the cost and prevent the application of the proposed system outside laboratories. However, we can expect that new sensors-which can work at close range and which can be made inexpensive-may solve this problem. The focus of this paper is on the analysis and design of variable admittance controllers. However, in the near future, we will substitute the LRFs with new, inexpensive and close-range sensors.

\section{Conclusions}

In this paper, we studied the pHRI between a human user and a mobile robot. We developed a robotic system which can measure a human's chest/ankle positions using four LRFs. The pHRI dynamics using admittance controllers in the global, robot and human frames were analysed and compared. With the LRF measurements, we could implement human frame admittance control to remove the nonlinearity in the system dynamics, and derive a stability criterion considering the effects of the PD controller and time discretization. By using an improved stiffness estimation method along with the derived stability criterion, a stiffness-based variable admittance controller was designed. The proposed method for improving the pHRI

5 We can observe that the right-hand side of (11) is an odd-ordered polynomial, which has a similar form to that of the example system discussed in [39]. The Lyapunov function for such a system might be non-polynomial and it demands non-trivial effort. 
performance is supported by simulations and experimental results.

The present work can be improved in several ways. First, the Lyapunov function corresponding to the admittance control in the robot frame is to be found. Besides, the current ankle velocity compensation method (SDVAC-C) was based on heuristics. To improve this method, a quantitative analysis and further comparison experiments with existing methods [27, 30, 32] are needed. Moreover, the current assumption as to the diagonal stiffness matrix and the estimation method can be improved by the methods in [27]. Finally, although the reduction of the interaction force using variable admittance control has been well-supported by the existing literature, more subjects should be invited in the experiments to test the potential instability in more complicated tasks (e.g., in pHRI closer approximating dancing with additional DOFs in the human's shoulders and pelvis). We will develop a new prototype to experimentally evaluate the performance of SDVAC in such complicated pHRI tasks [42].

\section{References}

[1] Sami Haddadin, Alin Albu-Schäffer, and Gerd Hirzinger. Safe physical human-robot interaction: Measurements, analysis and new insights. In Makoto Kaneko and Yoshihiko Nakamura, editors, Robotics Research, volume 66 of Springer Tracts in Advanced Robotics, pages 395-407. Springer Berlin / Heidelberg, 2011.

[2] Homayoon Kazerooni. Human-robot interaction via the transfer of power and information signals. IEEE Transactions on Systems, Man and Cybernetics, 20(2):450-463, 1990.

[3] H Kazerooni and Jenhwa Guo. Human extenders. Journal of Dynamic Systems, Measurement, and Control, 115(2B):281-290, 1993.

[4] Kazuhiro Kosuge, Yoshio Fujisawa, and Toshio Fukuda. Control of robot directly maneuvered by operator. In Proceedings of the IEEE/RSJ International Conference on Intelligent Robots and Systems, pages 49-54, 1993.

[5] Alin Albu-Schäffer, Christian Ott, and Gerd Hirzinger. A unified passivity-based control framework for position, torque and impedance control of flexible joint robots. The International Journal of Robotics Research, 26(1):23-39, 2007.

[6] Nicolas Lauzier, Martin Grenier, and Clément Gosselin. 2 DOF cartesian force limiting device for safe physical human-robot interaction. In Proceedings of the IEEE International Conference on Robotics and Automation, pages 253-258, 2009.

[7] Martin JD Otis, Vincent Duchaine, Greg Billette, Simon Perreault, Clément Gosselin, and Denis Laurendeau. Cartesian control of a cable-driven haptic mechanism. Advances in Haptics, pages 75102, 2010.

[8] Wei Yang Ho, Werner Kraus, Alexander Mangold, and Andreas Pott. Haptic interaction with a cabledriven parallel robot using admittance control. In Cable-Driven Parallel Robots, pages 201-212. Springer, 2015.

[9] Michael Peshkin and J Edward Colgate. Cobots. Industrial Robot: An International Journal, 26(5):335341, 1999.

[10] Yasuhisa Hirata, Asami Hara, and Kazuhiro Kosuge. Motion control of passive intelligent walker using servo brakes. IEEE Transactions on Robotics, 23(5):981-990, 2007.

[11] Joe Sanford, Isura Ranatunga, and Dan Popa. Physical human-robot interaction with a mobile manipulator through pressure sensitive robot skin. In Proceedings of the 6th International Conference on Pervasive Technologies Related to Assistive Environments, page 60. ACM, 2013.

[12] Samuel Agus Setiawan, Jin'ichi Yamaguchi, SangHo Hyon, and Atsuo Takanishi. Physical interaction between human and a bipedal humanoid robotrealization of human-follow walking. In Proceedings of the IEEE International Conference on Robotics and Automation, pages 361-367, 1999.

[13] Federico Augugliaro and Raffaello D'Andrea. Admittance control for physical human-quadrocopter interaction. In European Control Conference, pages 1805-1810, 2013.

[14] Prasad Akella, Michael Peshkin, ED Colgate, Nidamaluri Nagesh, Jim Wells, Steve Holland, Tom Pearson, and Brian Peacock. Cobots for the automobile assembly line. In Proceedings of the IEEE International Conference on Robotics and Automation, pages 728-733, 1999.

[15] Oscar Chuy, Yasuhisa Hirata, and Kazuhiro Kosuge. A new control approach for a robotic walking support system in adapting user characteristics. IEEE Transactions on Systems, Man, and Cybernetics, Part C: Applications and Reviews, 36(6): 725-733, 2006.

[16] O. Khatib, O. Brock, K. Yokoi, and R. Holmberg. Dancing with juliet 1999. In IEEE Robotics and Automation Conference Video Proceedings, 1999.

[17] Takahiro Takeda, Yasuhisa Hirata, and Kazuhiro Kosuge. Dance Partner Robot Cooperative Motion Generation with Adjustable Length of Dance Step Stride based on Physical Interaction. Proceedings of IEEE/RSJ International Conference on Intelligent Robots and Systems, pages 3258-3263, 2007.

[18] Neville Hogan. Impedance control: An approach to manipulation: Part i-theory. ASME Journal of Dynamic Systems, Measurement, and Control, 107:1-7, 1985. 
[19] Toru Tsumugiwa, Ryuichi Yokogawa, and Kei Hara. Variable impedance control based on estimation of human arm stiffness for human-robot cooperative calligraphic task. Proceedings of IEEE International Conference on Robotics and Automation, 1:644-650, 2002.

[20] Thomas H Massie and J Kenneth Salisbury. The phantom haptic interface: a device for probing virtual objects. Proceedings of the ASME Winter Annual Meeting, Symposium on Haptic Interfaces for Virtual Environment and Teleoperator Systems, 55(1): 295-300, 1994.

[21] Christian Ott, Ranjan Mukherjee, and Yoshihiko Nakamura. Unified impedance and admittance control. In Proceedings of the IEEE International Conference on Robotics and Automation, pages 554561, 2010.

[22] CL Clover, Greg R Luecke, James J Troy, and William A McNeely. Dynamic simulation of virtual mechanisms with haptic feedback using industrial robotics equipment. In Proceedings of the IEEE International Conference on Robotics and Automation, volume 1, pages 724-730, 1997.

[23] Kazuhiro Kosuge and Norihide Kazamura. Control of a robot handling an object in cooperation with a human. In Proceedings of the 6th IEEE International Workshop on Robot and Human Communication, pages 142-147, 1997.

[24] Russell Taylor, Pat Jensen, Louis Whitcomb, Aaron Barnes, Rajesh Kumar, Dan Stoianovici, Puneet Gupta, ZhengXian Wang, Eugene Dejuan, and Louis Kavoussi. A steady-hand robotic system for microsurgical augmentation. The International Journal of Robotics Research, 18(12):1201-1210, 1999.

[25] Matthew Spenko, Haoyong Yu, and Steven Dubowsky. Robotic personal aids for mobility and monitoring for the elderly. IEEE Transactions on Neural Systems and Rehabilitation Engineering, 14(3):344351, 2006.

[26] Vincent Duchaine and Clement M Gosselin. Investigation of human-robot interaction stability using Lyapunov theory. Proceedings of the IEEE International Conference on Robotics and Automation, pages 2189-2194, 2008.

[27] Vincent Duchaine and Clément Gosselin. Safe, stable and intuitive control for physical humanrobot interaction. Proceedings of the IEEE International Conference on Robotics and Automation, pages 3383 3388, 2009.

[28] Keng Peng Tee, Rui Yan, and Haizhou Li. Adaptive admittance control of a robot manipulator under task space constraint. In Proceedings of the IEEE International Conference on Robotics and Automation, pages 5181-5186, 2010.

[29] Vladislav Okunev, Thomas Nierhoff, and Sandra Hirche. Human-preference-based control design:
Adaptive robot admittance control for physical human-robot interaction. In Proceedings of the IEEE International Symposium on Robot and Human Interactive Communication, pages 443-448, 2012.

[30] Alexandre Lecours, Boris Mayer-St-Onge, and Clément Gosselin. Variable admittance control of a four-degree-of-freedom intelligent assist device. In Proceedings of the IEEE International Conference on Robotics and Automation, pages 3903-3908. IEEE, 2012.

[31] Clément Gosselin, Thierry Laliberté, Boris MayerSt-Onge, Simon Foucault, Alexandre Lecours, Vincent Duchaine, Noémie Paradis, Dalong Gao, and Roland Menassa. A friendly beast of burden: A human-assistive robot for handling large payloads. IEEE Robotics \& Automation Magazine, 20(4):139-147, 2013.

[32] Kohei Wakita, Jian Huang, Pei Di, Kosuke Sekiyama, and Toshio Fukuda. Human-walking-intention-based motion control of an omnidirectionaltype cane robot. IEEE/ASME Transactions on Mechatronics, 18(1):285-296, 2013.

[33] Fanny Ficuciello, Luigi Villani, and Bruno Siciliano. Variable impedance control of redundant manipulators for intuitive human-robot physical interaction. IEEE Transactions on Robotics, 31(4):850-863, 2015.

[34] R Brent Gillespie and Mark R Cutkosky. Stable userspecific haptic rendering of the virtual wall. Proceedings of the ASME International Mechanical Engineering Congress and Exhibition, 58:397-406, 1996.

[35] Angelika Peer. Design and Control of Admittance-Type Telemanipulation Systems. PhD thesis, Technische Universität München, 2008.

[36] Homayoun Seraji. Adaptive admittance control: an approach to explicit force control in compliant motion. In Proceedings of the IEEE International Conference on Robotics and Automation, pages 27052712, 1994.

[37] Vincent Duchaine, Boris Mayer St-Onge, Dalong Gao, and Clément Gosselin. Stable and intuitive control of an intelligent assist device. IEEE Transactions on Haptics, 5(2):148-159, 2012.

[38] RC Bonitz and Tien C Hsia. Internal force-based impedance control for cooperating manipulators. IEEE Transactions on Robotics and Automation, 12(1): 78-89, 1996.

[39] Amir Ali Ahmadi, Miroslav Krstic, and Pablo A. Parrilo. A globally asymptotically stable polynomial vector field with no polynomial lyapunov function. In IEEE Conference on Decision and Control and European Control Conference, pages 7579-7580, 2011.

[40] Hongbo Wang and Kazuhiro Kosuge. Understanding and reproducing waltz dancers' body dynamics 
in physical human-robot interaction. In Proceedings of the IEEE International Conference on Robotics and Automation, pages 3134-3140, 2012.

[41] Hongbo Wang and Kazuhiro Kosuge. Control of a robot dancer for enhancing haptic human-robot interaction in waltz. IEEE Transactions on Haptics, 5(3):264-273, 2012.
[42] Diego Felipe Paez Granados and Kazuhiro Kosuge. Design of a male-type dance partner robot for leading a physical human-robot interaction. In Proceedings of the IEEE International Conference on Mechatronics and Automation, 2015. To appear. 